\title{
Exosomes: the key of sophisticated cell- cell communication and targeted metastasis in pancreatic cancer
}

\author{
Huan Zhang ${ }^{1} \mathbb{D}$, Juan Xing ${ }^{1}$, Zhujiang Dai ${ }^{1}$, Daorong Wang ${ }^{2}$ and Dong Tang ${ }^{2 *}$
}

\begin{abstract}
Pancreatic cancer is one of the most common malignancies. Unfortunately, the lack of effective methods of treatment and diagnosis has led to poor prognosis coupled with a very high mortality rate. So far, the pathogenesis and progression mechanisms of pancreatic cancer have been poorly characterized. Exosomes are small vesicles secreted by most cells, contain lipids, proteins, and nucleic acids, and are involved in diverse functions such as intercellular communications, biological processes, and cell signaling. In pancreatic cancer, exosomes are enriched with multiple signaling molecules that mediate intercellular communication with control of immune suppression, mutual promotion between pancreas stellate cells and pancreatic cancer cells, and reprogramming of normal cells. In addition, exosomes can regulate the pancreatic cancer microenvironment and promote the growth and survival of pancreatic cancer. Exosomes can also build pre-metastatic micro-ecological niches and facilitate the targeting of pancreatic cancer. The ability of exosomes to load cargo and target allows them to be of great clinical value as a biomarker mediator for targeted drugs in pancreatic cancer.
\end{abstract}

Keywords: Pancreatic cancer (PaCa), Exosome, Cell-cell communication, Metastasis, Biomaker

\section{Background}

Pancreatic cancer $(\mathrm{PaCa})$ is the fourth leading cause of cancer mortality in the United States, with an overall 5 -year survival rate of $5 \%$ to $15 \%$ in the United States. Although surgical resection is the only potentially curative therapy, only $20 \%$ of $\mathrm{PaCa}$ are eligible for surgical resection at the time of diagnosis due to the rapid progression of $\mathrm{PaCa}$ and lack of early diagnostic access $[1,2]$. So, early diagnosis, prevention of metastasis, and effective treatment methods of pancreatic cancer are critical issues that need to be addressed [3]. The PaCa microenvironment, consisting of cancer cells, stromal cells, and extracellular matrix, is critical to the growth and survival

\footnotetext{
*Correspondence: 2179548523@qq.com

${ }^{2}$ Department of General Surgery, Institute of General Surgery, Northern Jiangsu People's Hospital, Clinical Medical College, Yangzhou University, Yangzhou 225001, China

Full list of author information is available at the end of the article
}

of $\mathrm{PaCa}$. The stromal cells that contribute to $\mathrm{PaCa}$ progression are mainly pancreatic stellate cells (PSCs), regulatory $\mathrm{T}$ cells (Tregs), myeloid-derived suppressor cells (MDSCs), and tumor-associated macrophages (TAMs). Besides, these cancerous cells can secrete extracellular components such as extracellular matrix (ECM), growth factors, and transforming growth factor-beta (TGF $\beta$ ), thereby creating a microenvironment that supports the survival and progression of $\mathrm{PaCa}$ [4]. Exosomes are a pathway for the above cells to secrete extracellular components and transport metastatic signals, significantly impacting the $\mathrm{PaCa}$ microenvironment and the normal cell microenvironment.

Exosomes are extracellular vesicles $40-150 \mathrm{~nm}$ in diameter that all cells can release. They consist of a lipid bilayer surrounding a small cytoplasm without cellular organelles, allowing for local and systematic transfer of intercellular components [5]. Current studies have shown 
that exosomes can be synthesized by different types of cells and released into the extracellular environment, promoting $\mathrm{PaCa}$ proliferation, angiogenesis, invasion of adjacent normal tissue structures, distant metastasis, and the occurrence of chemotherapy resistance. Since the discovery of exosomes in 1983, the utility of exosomes in $\mathrm{PaCa}$ has become increasingly significant [6]. This review focuses on the function of exosomal transport signals of $\mathrm{PaCa}$ origin, mediation of intercellular communication, and regulation of $\mathrm{PaCa}$ metastasis. Due to their stability and accumulation in the circulatory system, exosomes are enriched for many signaling molecules, making them clinical biomarkers for $\mathrm{PaCa}$. In addition, the ability of exosomes to act vectorially and bidirectionally have highlighted their clinical values in $\mathrm{PaCa}$ therapeutic research.

\section{Origin and structure of exosomes}

Exosome production begins with endocytosis, followed by a complex intracellular pathway, ending with cytosolic exocytosis (Fig. 1A). Altered cancer genotypes, phenotypes, endocytosis characteristics, and increased plasma membrane remodeling may lead to endocytosis. It has been shown in previous studies that cancer cells secrete more exosomes compared to normal cells
[5]. Early endosomes formed by embedding the plasma membrane can exchange and enrich signaling molecules with the cytoplasm, Golgi apparatus, and endoplasmic reticulum through mechanisms such as endosomal sorting complex required for transport (ESCRT), receptormediated internalization, and lipid interactions [7]. The "signaling endosome" determines the content and type of exosomal contents through cytoplasmic interactions [7]. The formation of later endosomes or multivesicular bodies (MVBs) from early endosomes has three known destinations: Firstly the direct secretion of contents into the endoplasmic reticulum or cytoplasm and the transGolgi network; secondly, phagocytosis and degradation by phagosomes, and finally fusion with the plasma membrane to secrete exosomes into the extracellular microenvironment (Fig. 1A) [8].

The secretion and release of exosomes into the extracellular microenvironment is dependent on several energetically active steps mediated by Rab GTPases, molecular motors, cytoskeletal proteins, and soluble N-ethylmaleimide-sensitive factor attachment protein receptors (SNAREs) [8, 9]. Previous studies demonstrated that plasmacytoma variant translocation 1 (PVT1) facilitates the transport of MVBs to the plasma membrane, thus

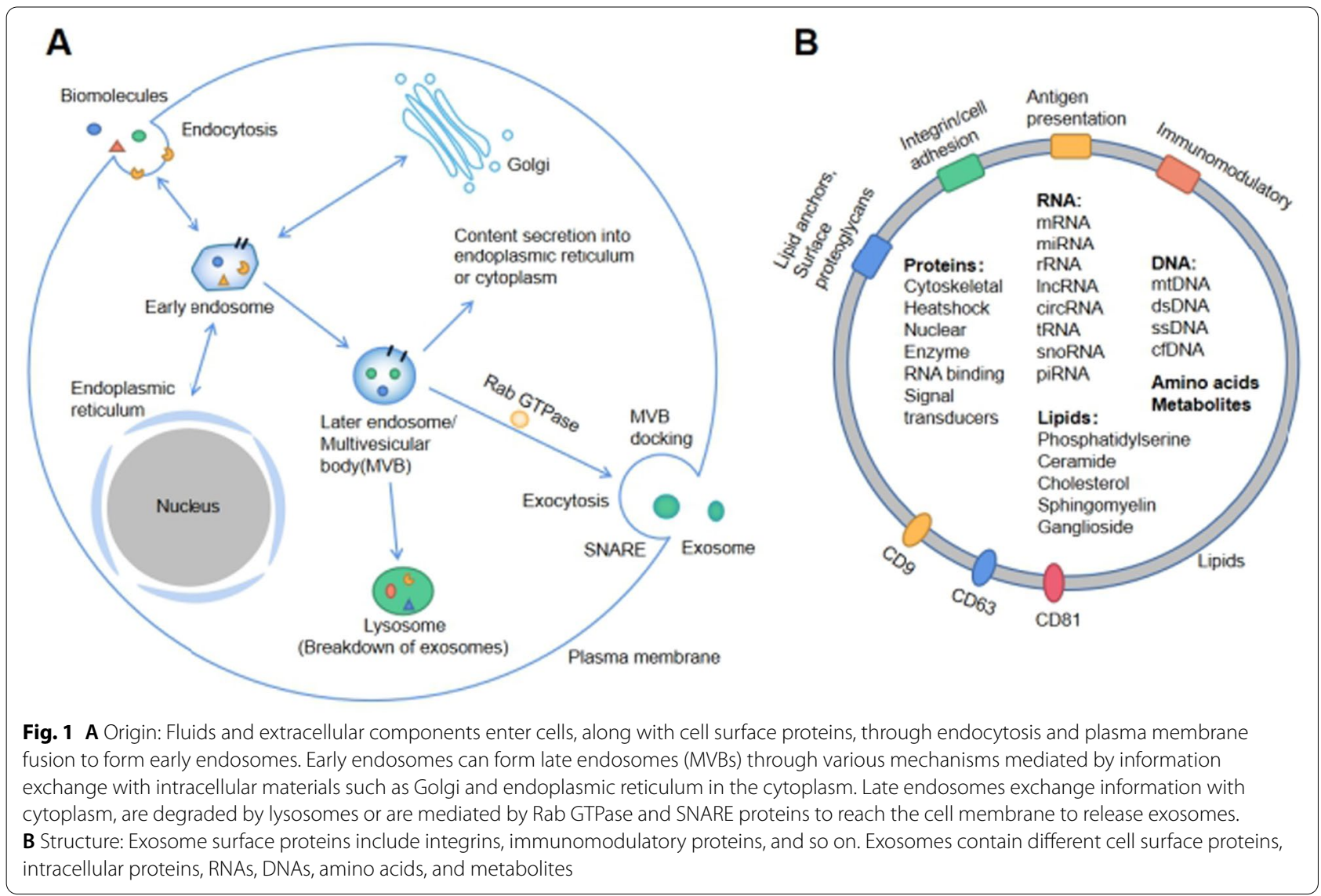


promoting the secretion of $\mathrm{PaCa}$-derived exosomes (Pex) [10]. The secreted exosomes can reach all body parts via the circulatory pathway and be taken up by specific organs, tissues, and cells. Exosomes can communicate with target cells through several known pathways: docking at the target cell's plasma membrane and activating intracellular signals through ligand-receptor interactions [8]. Another pathway is through fusion with the septum between the endocytic compartment through endocytosis and releasing its contents into the cytoplasm of the recipient cell, and lastly by absorption through membrane fusion and intracytoplasmic release.[8]

The lipid bilayer enveloping the exosome contains multiple signaling molecules, such as antigen-presenting molecules, integrins, surface proteoglycans, CD9, CD63, CD81, etc., which mediate exosome recognition and fusion, uptake and secretion (Fig. 1B). Exosomes carry a large number of cellular signaling molecules that regulate cell-cell communication, regulating the $\mathrm{PaCa}$ microenvironment in the proximity and later promoting $\mathrm{PaCa}$ invasion and metastasis. Exosomes can be modified to act as effective carriers for PaCa therapy and as a biomarker in PaCa liquid biopsies $[8,11,12]$.

\section{Exosomes mediate cell-cell communication in $\mathrm{PaCa}$}

$\mathrm{PaCa}$ is not an isolated entity but a network system involving cellular communication between malignant and normal cells [8]. Currently, widely accepted modes of cell-cell communication include (1) chemoreceptor-mediated contact, (2) direct cell-to-cell contact, and (3) cell -cell synaptic contact [13]. On the one hand, Pex secreted by $\mathrm{PaCa}$ can transport nucleic acids, proteins, and lipids from donor cells to recipient cells, releasing signals that induce inflammatory responses, suppress immune responses, regulate the antiapoptotic response of cancer cells and promote angiogenesis, thereby promoting cancer metastasis (Table 1); On the other hand, cells associated with $\mathrm{PaCa}$, such as tumor-associated macrophages (TAMs), cancer-initiating cells (CICs) and pancreatic stellate cells (PSCs), which release exosomes, can promote the growth, drug resistance, metastasis and invasion of $\mathrm{PaCa}$ (Fig. 2) $[8,10,11]$.

\section{Pex-mediated immune suppression of $\mathrm{PaCa}$}

A necessary condition for the survival and development of $\mathrm{PaCa}$ cells is the capacity to evade host immune surveillance [14]. Pex promotes the escape of immune surveillance by inhibiting the activation and survival of lymphocytes, thereby inducing a loss of lymphocyte function $[7,15,16]$.

$\mathrm{T}$ cells interact with Pex via the receptor/ligand signaling. In contrast, other lymphocytes (B cells, NK cells) and monocytes internalize Pex [17]. Pex can directly achieve immunosuppression by inducing apoptosis in activated anti-tumor effector cells or by polarising immune cells into a tumor-promoting phenotype. Pex can induce T cell apoptosis through pathways involving Fas ligand, TRAIL, and PDL- 1 receptor-mediated, $\mathrm{CD} 4{ }^{+} \mathrm{CD} 25^{+} \mathrm{Foxp} 3^{+}$Treg

Table 1 Potential exosomes biomarkers of $\mathrm{PaCa}$

\begin{tabular}{|c|c|c|c|}
\hline Biomarkers & Exosome function & Signaling pathway & References \\
\hline CD44v6 & Enhance migration and invasion & $\begin{array}{l}\text { Activate Wnt/ } \beta \text {-Catenin pathway and increase PAI-1, MMP } \\
\text { and TIM-1 }\end{array}$ & {$[44,95,96]$} \\
\hline Tspan8 & $\begin{array}{l}\text { Promote signaling, apoptosis-resistance, angiogenesis, } \\
\text { EMT, motility and invasion }\end{array}$ & Increase expression of chemokine and receptor & {$[44,97]$} \\
\hline MIF & Promote the formation of the liver pre-metastatic niche & $\begin{array}{l}\text { Up-regulate TGF- } \beta \text { expression, KC and HSC activation and induce } \\
\text { fibronectin secretion }\end{array}$ & {$[32]$} \\
\hline Clandin7 & Promote migration and invasion & $\begin{array}{l}\text { Increase pAkt/BCl-2/BCl-XL/MDR1, promote matrix degradation, } \\
\text { and reprogram SC and HPC }\end{array}$ & {$[48]$} \\
\hline $\operatorname{Lin} 28 B$ & Promote the recruitment of PSC & Activate Lin28B/let-7/HMGA2 /PDGFB axis & {$[52]$} \\
\hline GPC1 & Inform pancreas cancer burden & Carry mutant Kras mRNA and HS modifications & {$[98-100]$} \\
\hline C1QBP & HSC activation and liver fibrosis & Induce phosphorylation of the IGF-1 signaling molecule & {$[97]$} \\
\hline Plectin & Induce migration, proliferation and invasion & Not mentioned & {$[80]$} \\
\hline miR-1246 & Promote PSC proliferation and pancreatic fibrosis & $\begin{array}{l}\text { Induce Akt/ERK activation and increase a-SMA and procollagen } \\
\text { type I C-peptide }\end{array}$ & {$[51,97,101]$} \\
\hline miR-301a-3p & Promote metastasis and EMT & Mediate M2 macrophage polarization via PTEN/PI3Ky & {$[102]$} \\
\hline SRSF1 & Regulate exosome microRNA enrichment & Direct binding to miR-1246 sequence & {$[86]$} \\
\hline Inc-Sox2ot & Promote EMT and stem cell like properties & Bind to the miR-200 family & {$[101]$} \\
\hline ITGs & Promote tumor-specific metastasis & Promote adhesion and activate genes & {$[33]$} \\
\hline AEP & Enhance invasion and promote metastasis & Regulate activation of PI3K/AKT signaling & {$[102]$} \\
\hline CKAP4 & Proliferation and migration of PDAC cells & DKK1-dependent endocytosis routes & [103] \\
\hline
\end{tabular}




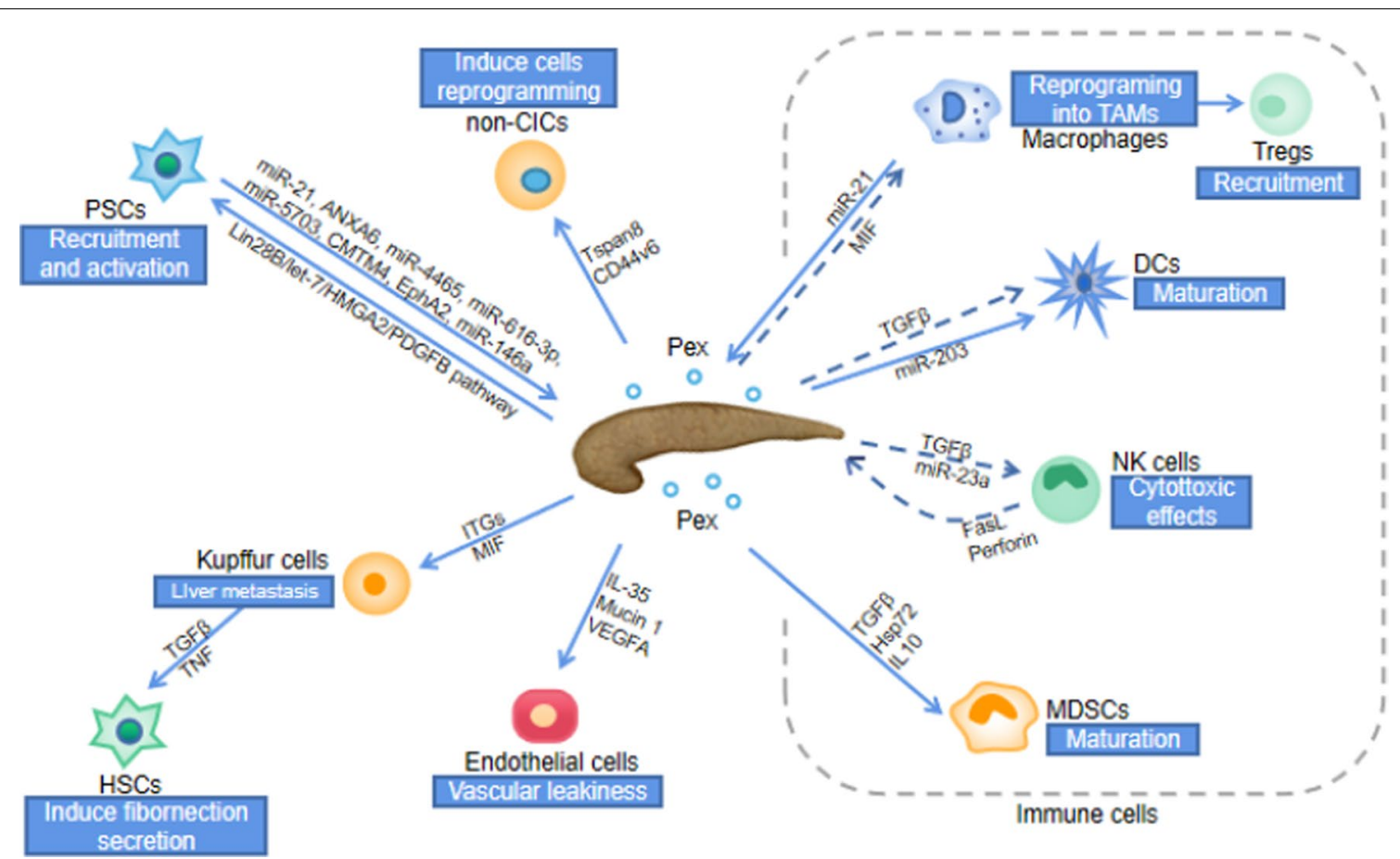

Fig. 2 Pex mediates cell-cell communication. CICs lead to reprogramming of non-CICs by secreting Pex. The preferential fusion of Pex with Kupffer cells leads to the recruitment and activation of HSCs, the formation of a fibrotic microenvironment and ultimately to liver metastasis. PaCa activates PSCs by secreting exosomes, and activated PSCs promote PaCa proliferation, metastasis and drug resistance by secreting exosomes. In terms of immune cells, Pex leads to reprogramming of macrophages, recruitment of Tregs, inhibition of NK cells, maturation of MDSCs through signalling molecules and ultimately to immune suppression. (dashed lines: inhibit, solid lines: promote)

cells [18-20]. Moreover, Pex inhibits the proliferation of $\mathrm{CD} 8^{+} \mathrm{T}$ cells but promotes the expansion of $\mathrm{CD} 4^{+}$ $T$ cells, particularly Treg [17]. Pex increases the secretion of proinflammatory cytokines IL-1 $\beta$, IL-6, and IL-10 while inhibiting the release of IFN $\gamma$, IL-2, and IL-17 from $\mathrm{CD}^{+}$and $\mathrm{CD} 8^{+} \mathrm{T}$ cells. This inhibits $\mathrm{T}$ cell proliferation and differentiation to Th1 and Th17 cells and promotes the production of Treg cells [8]. Macrophages are usually divided into two functional polarisation states: M1, which kills tumor cells, and M2, which promotes tumor growth [21]. It has been shown that exosomes modified by plasmid DNA expressing miR-155 or miR-125b-2 can lead to macrophage reprogramming in the pancreatic tumor microenvironment, leading to a potential pathway to inhibit $\mathrm{PaCa}$ invasion and metastasis [22]. In the tumor microenvironment, exosomes mediate the activation of macrophages into TAM, which exhibits M2 features [23]. TAM can further induce immune regulation, inflammatory responses, angiogenesis, and the establishment of a pre-metastatic microenvironment, thereby promoting tumor initiation and progression, invasion, and metastasis [24].

On the other hand, Pex can indirectly achieve immunosuppression by inhibiting functions necessary to maintain immunogenic responses such as activation, proliferation, and cytotoxicity. Pex deliver receptor-mediated signals to $\mathrm{T}$ cells that initiate sustained $\mathrm{Ca}^{2+}$ flux, resulting in subsequent activation of the relevant downstream pathways, alterations in the recipient cell transcriptome and ultimately translate into modified functional responses [17, 25]. In activated $\mathrm{CD} 4^{+} \mathrm{T}$ cells, STAT 5 phosphorylation is increased but is decreased in activated $\mathrm{CD} 8^{+} \mathrm{T}$ cells [15]. Adenosine generated from ATP by Pex converts activated B cells into regulatory B cells through CD39 and CD73, resulting in its inhibition [26]. Dendritic cells act as specialized antigen-presenting cells, influencing primary $\mathrm{T}$ cell response and achieving an anti-tumor cell response [27]. Pex can increase the levels of lncRNA and asparaginyl endopeptidase (AEP/legumain) mRNA in dendritic cells [28]. It can also indirectly amplify the proliferation of Treg and myeloid-derived suppressor cells (MDSCs) and upregulate their suppressive activity. This overall response contributes to tumor-induced immunosuppression and tumor immune escape [29,30]. TAM induces an inflammatory response and is accepted by resident macrophages in the liver. High macrophage migration inhibitory factor (MIF) levels within exosomes of $\mathrm{PaCa}$ origin can induce upregulation of secreted 
factors through exosomal integrin $\alpha \mathrm{V} \beta 5$ : TGF $\beta$ is associated with liver fibrosis. Furthermore, proinflammatory genes such as S100A8 and S100P are linked with metastasis [31-34]. It has been shown in previous studies that TAM-derived exosomal miR-501-3p can promote $\mathrm{PaCa}$ development through activation of the TGF- $\beta$ signaling pathway and is a novel molecular target for $\mathrm{PaCa}$ immunotherapy [35]. In addition, exosomal miRNAs have been shown to induce immune tolerance and promote metastasis and invasion of tumor cells [36]. Enhancing immune activity and activating anti-tumor immune cells by isolating exosomal proteins and missing exosomal miRNAs also provides a pathway for $\mathrm{PaCa}$ treatment [37]. In short, Pex can mediate immune suppression indirectly or directly by inducing apoptosis of anti-tumor effector cells, inducing polarization of immune cells to a tumorpromoting phenotype with inhibition of the anti-tumor response.

\section{Reprogramming of non-CICs initiates metastasis potential of $\mathrm{PaCa}$}

Cancer-initiating cells (CICs) plays a vital role in the initiation of $\mathrm{PaCa}$ migration and metastasis [38, 39]. At the cellular level, CIC-derived exosomes can transfer certain features of CICs into non-cancer-initiating cells (non-CICs), thereby inducing their reprogramming and promoting their transformational features, such as non-anchored growth, apoptosis resistance, migration, and invasion, until they are phenotypically modified as CICs. At the level of $\mathrm{PaCa}, \mathrm{CICs}$ induce tumor-stromal reorganization, stimulate angiogenesis, and promote immunosuppression of hematopoietic cells by producing exosomes resisting apoptosis. This results in a pre-metastatic ecological niche in the distal metastatic organ [11, $39,40]$.

CIC-derived exosomes use CD44 variant isoform 6 $(\mathrm{CD} 44 \mathrm{v} 6)$ and Tspan8 as hubs to regulate miRNA production and function, leading to the reprogramming of non-CICs. Tspans belong to a family of 4 transmembrane proteins, consisting mainly of CD9, CD63, CD81, CD82, CD53, and CD37, and are 100 times more enriched in exosomes than in their source cells [41]. CD44v6 is Tspan8-associated in tetraspanin-enriched microdomains (TEM) biogenesis and targeting, and therefore promotes Tspan8 transcription [42-44]. Depending on cellular requirements, TEM may selectively recruit membrane-associated proteins such as integrins, proteases, and other relevant signaling molecules, thus acting as a specific signaling platform [41]. CICs-derived exosomes, acting as hub initiating non-CICs activation, is centrally shaped by CD44v6 and profit from message delivery by Tspan8 [42]. Upon uptake of PaCa-derived CD44v6positive exosomes by other $\mathrm{PaCa}$ cells, they activate
Wnt $/ \beta$-Catenin signaling and upregulate the expression of plasminogen activator inhibitor 1, MMP, and tissue inhibitor of metalloproteases 1 . This enhances $\mathrm{PaCa}$ cells migration and metastasis [44]. CD44v6 activity in $\mathrm{PaCa}$ relies on the association with receptor tyrosine kinases (RTK) and is engaged in Wnt signaling via associated LDL receptor related protein 6 (LRP6), which promotes catenin pathway activation [45-47]. Studies have demonstrated that RTK inhibitors can neutralize tumor progression by CICs-derived exosomes and may be an effective therapeutic approach to defeat $\mathrm{CIC}$-derived exosomes [48]. PaCa exosomal Tspan8 may promote matrix degradation and reprogramming of the stroma and hematopoietic cells, which are essential steps for $\mathrm{PaCa}$ metastasis [11]. Blockade of exosome binding by anti-Tspan 8 blockers inhibits CICs-derived exosomes in promoting $\mathrm{PaCa}$ progression and mitigates their deleterious effects on non-CICs [42]. In addition, the expression of Tspan8 and other CICs marker proteins, such as integrin $\alpha 6 \beta 1$, CD104, EpCAM, and CXCR4, is also mutually regulated in PaCa cells $[11,43]$. So, with CD44v6 and Tspan8 acting as hubs, CICs-derived exosomes can induce non-CICs reprogramming, thereby inducing matrix reorganization and forming a cascade of pre-metastatic ecological for and initiating cancer metastasis.

\section{Pex mediate mutual promotion between PSCs and $\mathrm{PaCa}$ cells}

Pancreas stellate cells (PSC, also known as cancer-associated fibroblasts (CAF)) plays a crucial role in the development of chronic pancreatitis, pancreatic fibrosis, and cancerous environment $[49,50]$. Pex can induce PSC activation and pro-fibrosis, leading to a microenvironment of pre-cancerous fibrosis and the development of $\mathrm{PaCa}$ [51].

Pex can transfer Lin28B to PaCa cells, facilitating their recruitment and activation of PSCs via the Lin28B/ let-7/HMGA2/PDGFB pathway [52]. Activation of PSC is characterized by the secretion of exosomes. Exosomal miRNAs are key messengers for communication between PSC and PaCa cells [53]. PSC-derived exosomal miR-21 promotes $\mathrm{PaCa}$ cell migration and EMT and enhances Ras/ERK and Ras/Akt signaling pathway activity in pancreatic ductal carcinoma cells [54]. PSC-derived exosomes can induce chemoresistance by transferring miR-21 into cancer cells, binding apoptosis peptidaseactivating factor 1 (APAF1) or activating the phosphatidylinositol 3-kinase (PI3K)/Akt signaling pathway [55]. Downregulation of miR-21 was shown to inhibit the migration and invasion of PSC [56]. Besides, PSCderived exosomes can also transmit chemoresistance by regulating the expression levels of EphA2, miR-146a and Snail signaling cascade in $\mathrm{PaCa}[57,58]$. Proliferation 
and metastasis of $\mathrm{PaCa}$ are promoted by regulating the expression levels of ANXA6, miR-4465, miR-616-3p, miR-5703 and CMTM4 [59-61]. PSCs contribute significantly to the chemoresistant nature of $\mathrm{PaCa}$ by producing extensive fibrous ECM, which results in (1) altered vasculature and decreased drug delivery to the tumor; (2) Reduced sensitivity to chemotherapeutic agents; and (3) increased epithelial-mesenchymal transition [62]. Hence, downregulation of exosomal miRNA or inhibition of exosomal secretion can inhibit the function of PSC, thus suppressing the occurrence, development and metastasis of PaCa. The exosomal inhibitor GW4869 inhibits the stimulatory effects of PSC on proliferation, migration and chemokine gene expression [63]. Pex is involved in PSC recruitment and activation, and activated PSC leads to proliferation, metastasis and chemoresistance of $\mathrm{PaCa}$ by secreting the exosome exosomal miRNAs. This bidirectional interaction between PSC and malignant cells, delivered by the exosome, favours tumour progression and metastasis [62].

\section{Exosomes induce targeted metastasis of $\mathrm{PaCa}$}

The extremely rapid progression, invasion and metastasis of pancreatic cancer leads to a very poor prognosis and high mortality rate. In addition to their impact on the local tumor microenvironment, exosomes also mediate distant cell-cell communication. They play an essential role in creating the pre-metastatic microenvironment of $\mathrm{PaCa}$ with invasion and metastasis through the regulation of fibroblast activation, ECM production, angiogenesis and immune surveillance.

Exosomes are critical determinants of organ-specific metastasis in PaCa through the expression levels of different integrins (ITG) [33, 64]. ITG can determine organotropic metastasis by fusing with organ-specific resident cells to establish pre-metastatic niche through activating Src phosphorylation and proinflammatory S100 expression $[33,65]$. The organs most susceptible to metastasis from $\mathrm{PaCa}$ are the liver and lung. In $\mathrm{PaCa}$ liver metastasis, exosomes expressing ITG $\alpha v \beta 5$ specifically bind to liver kupffer cells, Pex are transported to the liver via the humoral route fuses with kupffer cells (Fig. 3) [33]. The exosome-loaded MIF activates hepatic stellate cells, resulting in pro-fibrotic activity and remodeling of the extracellular matrix. Pro-fibrotic-related genes, such as TGF $\beta$ and TNF, are secreted by kupffer cells and increase liver metastasis burden [32]. Activated hepatic stellate cells participate in the inflammatory response, liver fibrosis and reconstruction of intrahepatic structures through proliferation and secretion of fibronectin. Intrahepatic sinusoidal pressure is increased through cellular contraction [32]. The resulting fibrotic microenvironment promotes the recruitment of bone marrow-derived cells that bind to fibronectinrich hepatic sites, eventually forming pre-metastatic sites

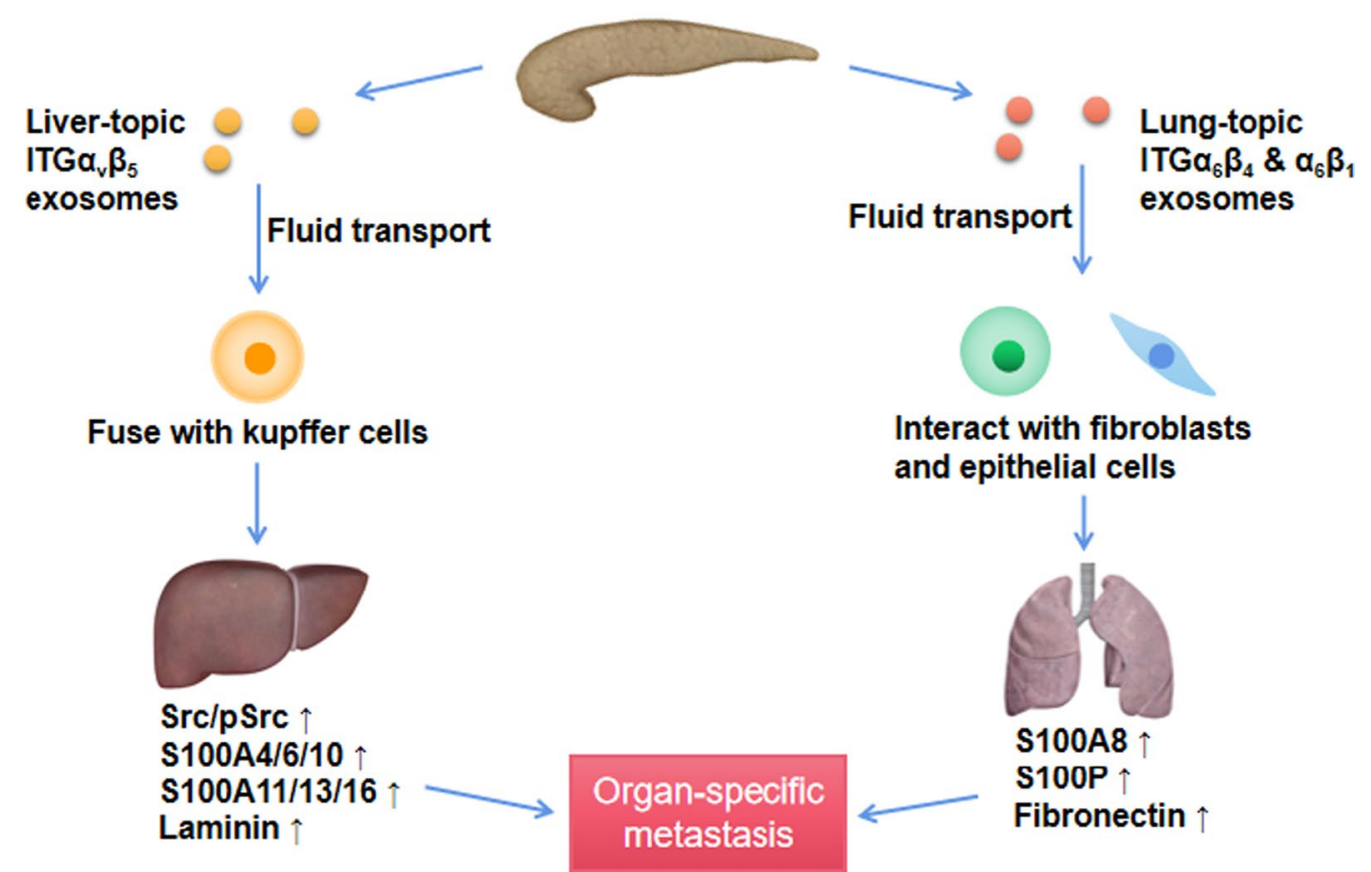

Fig. 3 Pex direct organ-specific metastasis via integrins. Pex can display different integrin proteins on their surface; ITGa $\beta_{4}$ - and ITGa $\beta_{1}$-expressing exosomes preferentially interact with fibroblasts and epithelial cells in lung, and ITGa $\beta_{5}$-expressing exosomes preferentially fuse with kupffer cells in liver. Once uptaken, Pex induce cellular changes in the target organ, thus promoting cancer cell colonization and organ-specific metastasis 
in the liver [32]. Furthermore, TAM acceptance by liver resident macrophages induces an inflammatory response, and high levels of MIF in the exocytotic body, mediated by ITG $\alpha \mathrm{V} \beta 5$, can induce upregulation of liver fibrosis-related factors and proinflammatory genes [32, 33]. In response to this inflammatory microenvironment, hepatic stellate cells are also activated, promoting liver metastasis and creating a vicious cycle. In order to access distant tissues, exosomes can disrupt endothelial cell junctions by increasing vascular permeability. Vesicles and cells enter the tissue parenchyma and alter the ECM, inducing thrombosis, creating a pre-metastatic microenvironment [66]. Increased VEGFA expression and serum concentrations in pancreatic cancer tumors are associated with worse prognosis and metastatic progression, particularly liver metastases [67]. In $\mathrm{PaCa}$, exosomes can regulate VEGF expression via STAT3, NF-kb, and Mucin 1 [68-70]. Lung-tropic exosomes expressing ITG $\alpha 6 \beta 4$ or ITG $\alpha 6 \beta 1$ can specifically interact with S100A4-positive fibroblasts and surfactant protein C-positive epithelial cells and determining $\mathrm{PaCa}$ lung metastasis [33, 64]. ITGs significantly upregulated proinflammatory S100 gene expression and increased Src or phosphorylated Src levels in resident cells, thus promoting cancer cell colonization and organ-specific metastasis [65].

Furthermore, after homing to their target tissue, Pex can play a role in the activation of a reactive, myofibroblast-rich stroma that promotes a host of tumor-supportive processes such as ECM remodeling, proliferation, and angiogenesis [64, 71-74]. For example, exosomal-transduced TGF $\beta /$ Smad signaling has been shown to underline the differentiation process $[64,73]$. Pex internalized by myofibroblast progenitors was shown to enhance their recruitment and trigger their differentiation into myofibroblast-like cells [64, 71-73]. By expressing immunomodulatory molecules such as CD39 and CD73, exosomes modulate host immunity, weakening immune effector cell responses and triggering immunosuppressive cells. This helps cancerous cells evade immune surveillance, allowing cancer progression and even generating a pre-metastatic microenvironment by activating an inflammatory response to promote completion [64].

In general, exosomes carry an abundance of pancreatic cancer signaling molecules closely associated with distant metastasis. These molecules determine the organ specificity of pancreatic cancer metastasis and contribute to establishing a pre-metastatic microenvironment.

\section{The clinical value of Pex: sources of biomarkers and drug carriers}

Traditional biomarkers such as carcinoembryonic antigen (CEA) and cancer antigen 19-9 (CA19-9) have improved the diagnostic accuracy of $\mathrm{PaCa}$, but they are less specific for PaCa [75]. Serous exosome concentrations tend to be higher in patients with $\mathrm{PaCa}$ than healthy controls. The load of exosomes varies according to the patient's health status, providing a powerful tool for PaCa biopsy [5]. A recent research hotspot in the field of exosome tools for $\mathrm{PaCa}$ research is surface-enhanced Raman scattering (SERS). Li TD et al. developed a PEARL SERS nano-tag that achieves ultra-sensitive and specific detection limits for Pex [75]. Pang Y et al. developed a DSN-assisted dual-SERS biosensor for direct quantification of target microRNAs in plasma-derived exosomes from pancreatic ductal adenocarcinoma (PDAC), chronic pancreatitis (CP) and normal controls (NC). They found significant SERS signal differences between these conditions [76]. In terms of cell surface markers, Pex can be enriched and carry many signaling molecules (Table 1). Glycosylation in cancer is considered a potential pathway for predicting cancer prognosis and exosomal glycan moieties are considered to be effective diagnostic markers in cancer detection [77, 78]. Glycan sequence analysis of exosomal subpopulations in PaCa cell lines and Pex detection techniques have been developed and reported for the study of exosomal glycan moieties. These techniques have also provided novel markers for PaCa [79, 80]. Fan J et al. isolated Pex from three $\mathrm{PaCa}$ cell lines that showed resistance to gemcitabine and reported that expression of exosome EphA2 could convey chemoresistance and predicted that it might be a powerful surface biomarker of exosomes [57, 81-83].

The ability of exosomes to be enriched with PaCa signaling molecules, mediate cell-cell communication, evade immune surveillance, bind specifically to target cells, and intermembranous transport show great promises as an area of research for drug delivery applications and therapeutic development [12, 84-86]. Kamerkar et al. show that exosomes from normal fibroblasts can be engineered to carry short interfering RNA or short hairpin RNA. These exosomes are more effective in targeting oncogenic kirsten rat sarcoma viral oncogene homolog (Kras) and inhibiting PaCa compared to liposomes [87]. McAndrews KM et al. show that engineered exosomes can serve as a delivery platform for CRISPR/Cas9 DNA to inhibit oncogenic Kras ${ }^{\mathrm{G} 12 \mathrm{D}}$ in vitro and suppress $\mathrm{PaCa}$ growth in vivo [88]. P21-activated kinase 4 (PAK4) is oncogenic when overexpressed, promoting cell survival, migration and unanchored growth [89, 90]. Using PAK4 as a therapeutic target in an in vivo mouse model with $\mathrm{PaCa}$ using exosome-mediated intra-tumor administration has been shown to reduce the growth of $\mathrm{PaCa}$ cells and improve survival rate in mice $(p<0.001)$ [91]. Pex exhibited the same efficacy and safety profile as polyethyleneimine in vivo RNAi transfection reagents, demonstrating their feasibility as drug carriers for use in $\mathrm{PaCa}$ therapy [91]. SRSF1 mediated the selective enrichment of 
exosomal miRNAs in PaCa cells by binding to the miR1246 sequence [85]. GAIP The deletion of the C-terminus of the interacting protein could lead to the upregulation of the autophagy marker LC3II, autophagy in PaCa cells. It could also control the cellular transport pathway by regulating exosomes' secretion, which subsequently determines the loading of cellular cargo in exosomes [92]. What's more, Zhou W et al. demonstrate an exosome-based dual delivery biosystem for enhancing PDAC immunotherapy as well as reversing tumor immunosuppression of M2-like tumor associated macrophages (M2-TAMs) upon disruption of galectin-9/dectin 1 axis [93]. The use of biomaterials, bone marrow mesenchymal stem cell exosomes, can significantly improve tumor targeting efficacy, thus increasing drug accumulation in the tumor site. Targeting of the tumor cells can also be improved by anchoring superparamagnetic nanoparticles to exosomes, which are "guided" towards the tumor by moderate magnetic fields [94].

In conclusion, a large number of studies have demonstrated that exosomes can be used as sources of biomarkers and drug carriers for $\mathrm{PaCa}$ treatment, and are of great value for pancreatic cancer diagnosis and treatment. However, the effectiveness of the above-mentioned markers and drug carrier functions is yet to be clinically validated. The complex biological properties of exosomes with selective loading mechanisms have not been thoroughly studied, which limits the progress of exosome research. How to achieve precise targeting of exosomes for gene editing is still an open question. Besides, we believe that targeting the metastatic signaling molecules on the surface of exosomes can make the target cells unable to recognize and accept exosomes, prevent the establishment of pre-metastatic microenvironment of pancreatic cancer and prevent invasive metastasis.

\section{Conclusion}

Pex produced by MVBs secretion carries many signaling molecules for $\mathrm{PaCa}$ and are versatile and critical intercellular messengers. Between neighboring cells, exosomes help $\mathrm{PaCa}$ achieve immune evasion, promote angiogenesis and foster fibrosis in the pancreatic microenvironment, thus creating a cancer microenvironment that promotes the survival and development of $\mathrm{PaCa}$. Between distal organ cells, exosomes are recognized and taken up by specific distal organ cells via humoral transport, creating a pre-metastatic ecological niche and stimulating distant metastasis. In the short 30 years since their discovery, exosomes have soared to the forefront of cell biology, cell signaling, cancer biology, immunology and drug delivery and have paved the way for clinical diagnostics and clinical therapeutic trials [7]. Exosomes are double-edged swords. If used by cancerous pancreatic cells, exosomes can shape the cancer microenvironment, creating a pre-metastatic ecological niche and facilitating its invasion and metastasis. If used therapeutically, exosomes can be a powerful aid to drug delivery and clinical treatment. The key, therefore, is how to use exosomes as a tool for our treatment. There are many unanswered questions about exosomes concerning their mode of transport, identification, and specific contents. The proper use of this therapeutic model requires further analysis and research of its specific clinical value.

\section{Abbreviations \\ AEP: Asparaginyl endopeptidase; CAFs: Cancer associated fibroblasts; CA19-9: Cancer antigen 19-9; CD44v6: CD44 variant isoform 6; CEA: Carcinoembryonic antigen; CICs: Cancer initiating cells; EMT: Epithelial-mesenchymal transition; ESCRT: Endosomal sorting complex required for transport; GPC1: Glypican-1; Kras: Kirsten rat sarcoma viral oncogene homolog; LRP6: LDL receptor related protein 6; MIF: Migration inhibitory factor; MVBs: Multivesicular bodies; non- CICs: Non-cancer-initiating cells; PaCa: Pancreatic cancer; PAK4: P21-activated kinase 4; PDAC: Pancreatic ductal adenocarcinoma; Pex: PaCa-derived exosomes; PSCs: Pancreatic stellate cells; RTK: Receptor tyrosine kinases; PVT1: Plasmacytoma variant translocation 1; SERS: Surface-enhanced Raman scat- tering; SNAREs: Soluble N-ethylmaleimide-sensitive factor attachment protein receptors; TAMs: Tumor-associated macrophages; TEM: Etraspanin-enriched microdomains; TGF $\beta$ : Transforming growth factor beta.}

\section{Acknowledgements}

Not applicable.

\section{Authors' contributions}

ZH drafted the manuscript and counted and plotted the diagram and tables. DT and DW critically revised the article for important intellectual content. All authors read and approved the final manuscript.

\section{Funding}

This work was supported by grants from the Training Project of Key Talents of Youth Medicine in Jiangsu province, China [No. QNRC2016330], the Key disease standardization diagnosis and treatment project in Jiangsu province [BE2015664], the Academic Science and Technology Innovation Fund for College Students [No. × 20180714], the Social Development-Health Care Project of Yangzhou, Jiangsu Province [No. YZ2018087], the Social Development Project of Yangzhou, Jiangsu Province [No. YZ2021075], and High-level talent "six one projects" top talent scientific research project of Jiangsu Province [No. LGY2019034]. The funding bodies had no role in writing the manuscript.

Availability of data and materials

Not applicable.

\section{Declarations}

Ethics approval and consent to participate

Not applicable.

\section{Consent for publication}

Not applicable.

\section{Competing interests}

The authors declare that they have no competing interests.

\section{Author details}

${ }^{1}$ Clinical Medical College, Yangzhou University, Yangzhou, Jiangsu Province, China. ${ }^{2}$ Department of General Surgery, Institute of General Surgery, Northern Jiangsu People's Hospital, Clinical Medical College, Yangzhou University, Yangzhou 225001, China. 
Received: 21 September 2021 Accepted: 21 November 2021

Published online: 15 January 2022

\section{References}

1. Puckett Y, Garfield K. Pancreatic cancer. In: StatPearls. Treasure Island (FL); 2021

2. Bisht S, Feldmann G. Novel targets in pancreatic cancer therapycurrent status and ongoing translational efforts. Oncol Res Treat. 2018:41:596-602.

3. Lan B, Zeng S, Grutzmann R, Pilarsky C. The role of exosomes in pancreatic cancer. Int J Mol Sci. 2019;20:1

4. Ren B, Cui M, Yang G, Wang H, Feng M, You L, Zhao Y. Tumor microenvironment participates in metastasis of pancreatic cancer. Mol Cancer. 2018;17:108

5. Kalluri R. The biology and function of exosomes in cancer. J Clin Invest. 2016;126:1208-15.

6. Harding CV, Heuser JE, Stahl PD. Exosomes: looking back three decades and into the future. J Cell Biol. 2013:200:367-71.

7. Milane L, Singh A, Mattheolabakis G, Suresh M, Amiji MM. Exosome mediated communication within the tumor microenvironment. J Control Release. 2015;219:278-94.

8. Maia J, Caja S, Strano Moraes MC, Couto N, Costa-Silva B. Exosomebased cell-cell communication in the tumor microenvironment. Front Cell Dev Biol. 2018:6:18

9. Pfeffer SR. Unsolved mysteries in membrane traffic. Annu Rev Biochem. 2007;76:629-45.

10. Sun C, Wang P, Dong W, Liu H, Sun J, Zhao L. LncRNA PVT1 promotes exosome secretion through YKT6, RAB7, and VAMP3 in pancreatic cancer. Aging. 2020;12:10427-40.

11. Sun W, Ren Y, Lu Z, Zhao X. The potential roles of exosomes in pancreatic cancer initiation and metastasis. Mol Cancer. 2020;19:135.

12 Kalluri R, LeBleu VS. The biology, function, and biomedical applications of exosomes. Science. 2020:367:1.

13. Valadi H, Ekstrom K, Bossios A, Sjostrand M, Lee JJ, Lotvall JO. Exosomemediated transfer of mRNAs and microRNAs is a novel mechanism of genetic exchange between cells. Nat Cell Biol. 2007;9:654-9.

14. Hanahan D, Folkman J. Patterns and emerging mechanisms of the angiogenic switch during tumorigenesis. Cell. 1996;86:353-64.

15. Whiteside TL. Immune modulation of T-cell and NK (natural killer) cell activities by TEXs (tumour-derived exosomes). Biochem Soc Trans. 2013:41:245-51.

16. Whiteside TL. Exosomes in cancer: another mechanism of tumorinduced immune suppression. Adv Exp Med Biol. 2017;1036:81-9.

17. Muller L, Mitsuhashi M, Simms P, Gooding WE, Whiteside TL. Tumorderived exosomes regulate expression of immune function-related genes in human T cell subsets. Sci Rep. 2016;6:20254.

18. Wieckowski EU, Visus C, Szajnik M, Szczepanski MJ, Storkus WJ, Whiteside TL. Tumor-derived microvesicles promote regulatory $T$ cell expansion and induce apoptosis in tumor-reactive activated CD8+ T lymphocytes. J Immunol. 2009;183:3720-30.

19. Abusamra AJ, Zhong Z, Zheng X, Li M, Ichim TE, Chin JL, Min WP. Tumor exosomes expressing Fas ligand mediate CD8+ T-cell apoptosis. Blood Cells Mol Dis. 2005:35:169-73.

20. Kim JW, Wieckowski E, Taylor DD, Reichert TE, Watkins S, Whiteside TL. Fas ligand-positive membranous vesicles isolated from sera of patients with oral cancer induce apoptosis of activated T lymphocytes. Clin Cancer Res. 2005:11:1010-20.

21. Martinez FO, Sica A, Mantovani A, Locati M. Macrophage activation and polarization. Front Biosci. 2008;13:453-61.

22. Su M, Aldawsari H, Amiji M. Pancreatic cancer cell exosome-mediated macrophage reprogramming and the role of MicroRNAs 155 and $125 \mathrm{~b} 2$ transfection using nanoparticle delivery systems. Sci Rep. 2016;6:30110.

23. Mantovani A, Sica A, Sozzani S, Allavena P, Vecchi A, Locati M. The chemokine system in diverse forms of macrophage activation and polarization. Trends Immunol. 2004;25:677-86.

24. Qian BZ, Pollard JW. Macrophage diversity enhances tumor progression and metastasis. Cell. 2010;141:39-51.

25. Muller L, Simms P, Hong CS, Nishimura MI, Jackson EK, Watkins SC, Whiteside TL. Human tumor-derived exosomes (TEX) regulate Treg functions via cell surface signaling rather than uptake mechanisms. Oncoimmunology. 2017;6:e1261243.

26. Figueiro F, Muller L, Funk S, Jackson EK, Battastini AM, Whiteside TL. Phenotypic and functional characteristics of CD39(high) human regulatory B cells (Breg). Oncoimmunology. 2016;5:e1082703.

27. Liu Y, Gu Y, Cao X. The exosomes in tumor immunity. Oncolmmunology. 2015;4:e1027472.

28. Chen L, Tan Y-Y, Chen Z-X, Wang T, Hu S, Nan Z-A, Xie L-Q, Hui Y, Huang J-X, Zhan C, et al. Toward long-term stability: single-crystal alloys of cesium-containing mixed cation and mixed halide perovskite. J Am Chem Soc. 2019;141:1665-71.

29. Szajnik M, Czystowska M, Szczepanski MJ, Mandapathil M, Whiteside TL. Tumor-derived microvesicles induce, expand and up-regulate biological activities of human regulatory $T$ cells (Treg). PLoS ONE. 2010;5:e11469

30. Xiang X, Poliakov A, Liu C, Liu Y, Deng ZB, Wang J, Cheng Z, Shah SV, Wang GJ, Zhang $L$, et al. Induction of myeloid-derived suppressor cells by tumor exosomes. Int J Cancer. 2009:124:2621-33.

31. Lukanidin E, Sleeman JP. Building the niche: the role of the $S 100$ proteins in metastatic growth. Semin Cancer Biol. 2012;22:216-25.

32. Costa-Silva B, Aiello NM, Ocean AJ, Singh S, Zhang H, Thakur BK, Becker A, Hoshino A, Mark MT, Molina H, et al. Pancreatic cancer exosomes initiate pre-metastatic niche formation in the liver. Nat Cell Biol. 2015;17:816-26.

33. Hoshino A, Costa-Silva B, Shen TL, Rodrigues G, Hashimoto A, Tesic Mark M, Molina H, Kohsaka S, Di Giannatale A, Ceder S, et al. Tumour exosome integrins determine organotropic metastasis. Nature. 2015;527:329-35

34. Matsushita H, Yang Y, Pandol S, Seki E. Exosome migration inhibitory factor as a marker and therapeutic target for pancreatic cancer. Gastroenterology. 2016;150:1033-5.

35. Yin Z, Ma T, Huang B, Lin L, Zhou Y, Yan J, Zou Y, Chen S. Macrophagederived exosomal microRNA-501-3p promotes progression of pancreatic ductal adenocarcinoma through the TGFBR3-mediated TGF-beta signaling pathway. J Exp Clin Cancer Res. 2019:38:310.

36. Azmi AS, Bao B, Sarkar FH. Exosomes in cancer development, metastasis, and drug resistance: a comprehensive review. Cancer Metastasis Rev. 2013:32:623-42.

37. Erb $U$, Zöller M. Progress and potential of exosome analysis for early pancreatic cancer detection. Expert Rev Mol Diagn. 2016:16:757-67.

38. Mimeault M, Batra SK. New advances on critical implications of tumor- and metastasis-initiating cells in cancer progression, treatment resistance and disease recurrence. Histol Histopathol. 2010;25:1057-73.

39. Zoller M. Exosomes in cancer disease. Methods Mol Biol. 2016:1381:111-49.

40. Salem KZ, Moschetta M, Sacco A, Imberti L, Rossi G, Ghobrial IM, Manier S, Roccaro AM. Exosomes in tumor angiogenesis. Methods Mol Biol. 2016:1464:25-34

41. Perez-Hernandez D, Gutierrez-Vazquez C, Jorge I, Lopez-Martin S, Ursa A, Sanchez-Madrid F, Vazquez J, Yanez-Mo M. The intracellular interactome of tetraspanin-enriched microdomains reveals their function as sorting machineries toward exosomes. J Biol Chem. 2013;288:11649-61.

42. Wang Z, Sun H, Provaznik J, Hackert T, Zöller M. Pancreatic cancerinitiating cell exosome message transfer into noncancer-initiating cells: the importance of CD44v6 in reprogramming. J Exp Clin Cancer Res CR. 2019:38:132.

43. Wang H, Rana S, Giese N, Buchler MW, Zoller M. Tspan8, CD44v6 and alpha6beta4 are biomarkers of migrating pancreatic cancer-initiating cells. Int J Cancer. 2013;133:416-26.

44. Wang Z, von Au A, Schnolzer M, Hackert T, Zoller M. CD44v6-competent tumor exosomes promote motility, invasion and cancer-initiating cell marker expression in pancreatic and colorectal cancer cells. Oncotarget. 2016;7:55409-36

45. Morath I, Jung C, Leveque R, Linfeng C, Toillon RA, Warth A, OrianRousseau V. Differential recruitment of CD44 isoforms by ErbB ligands reveals an involvement of CD44 in breast cancer. Oncogene. 2018:37:1472-84 
46. Schmitt M, Metzger M, Gradl D, Davidson G, Orian-Rousseau V. CD44 functions in Wnt signaling by regulating LRP6 localization and activation. Cell Death Differ. 2015;22:677-89.

47. Sun H, Rana S, Wang Z, Zhao K, Schnölzer M, Provaznik J, Hackert T, LV Q, Zöller M. The Pancreatic cancer-initiating cell marker CD44v6 affects transcription, translation, and signaling: consequences for exosome composition and delivery. J Oncol. 2019;2019:3516973.

48. Kyuno D, Zhao K, Schnölzer M, Provaznik J, Hackert T, Zöller M. Claudin7-dependent exosome-promoted reprogramming of nonmetastasizing tumor cells. Int J Cancer. 2019;145:2182-200.

49. Melstrom LG, Salazar MD, Diamond DJ. The pancreatic cancer microenvironment: a true double agent. J Surg Oncol. 2017;116:7-15.

50. Jin G, Hong W, Guo Y, Bai Y, Chen B. Molecular mechanism of pancreatic stellate cells activation in chronic pancreatitis and pancreatic cancer. J Cancer. 2020;11:1505-15.

51. Masamune A, Yoshida N, Hamada S, Takikawa T, Nabeshima T, Shimosegawa T. Exosomes derived from pancreatic cancer cells induce activation and profibrogenic activities in pancreatic stellate cells. Biochem Biophys Res Commun. 2018;495:71-7.

52. Zhang YF, Zhou YZ, Zhang B, Huang SF, Li PP, He XM, Cao GD, Kang MX, Dong X, Wu YL. Pancreatic cancer-derived exosomes promoted pancreatic stellate cells recruitment by pancreatic cancer. J Cancer. 2019;10:4397-407.

53. Lugea A, Waldron RT. Exosome-mediated intercellular communication between stellate cells and cancer cells in pancreatic ductal adenocarcinoma. Pancreas. 2017;46:1-4.

54. Ma QY, Zhang JQ, Lai Q, Zhang F, Dong ZH, A LS. Temporal and spatial variations of extreme climatic events in Songnen Grassland, Northeast China during 1960-2014. Ying Yong Sheng Tai Xue Bao. 2017;28:1769-78.

55. Au Yeung CL, Co NN, Tsuruga T, Yeung TL, Kwan SY, Leung CS, Li Y, Lu ES, Kwan K, Wong KK, et al. Exosomal transfer of stroma-derived miR21 confers paclitaxel resistance in ovarian cancer cells through targeting APAF1. Nat Commun. 2016;7:11150.

56. Ali S, Suresh R, Banerjee S, Bao B, Xu Z, Wilson J, Philip PA, Apte M, Sarkar $\mathrm{FH}$. Contribution of microRNAs in understanding the pancreatic tumor microenvironment involving cancer associated stellate and fibroblast cells. Am J Cancer Res. 2015:5:1251-64.

57. Fan J, Wei Q, Koay E, Liu Y, Ning B, Bernard P, Zhang N, Han H, Katz M, Zhao Z, Hu Y. Chemoresistance transmission via exosome-mediated EphA2 transfer in pancreatic cancer. Theranostics. 2018;8:5986-94.

58. Richards KE, Zeleniak AE, Fishel ML, Wu J, Littlepage LE, Hill R. Cancerassociated fibroblast exosomes regulate survival and proliferation of pancreatic cancer cells. Oncogene. 2016;36:1770-8.

59. Li M, Guo H, Wang Q, Chen K, Marko K, Tian X, Yang Y. Pancreatic stellate cells derived exosomal miR-5703 promotes pancreatic cancer by downregulating CMTM4 and activating PI3K/Akt pathway. Cancer Lett. 2020;490:20-30

60. Cao W, Zeng Z, He Z, Lei S. Hypoxic pancreatic stellate cell-derived exosomal mirnas promote proliferation and invasion of pancreatic cancer through the PTEN/AKT pathway. Aging (Albany NY). 2021;13:7120-32.

61. Leca J, Martinez S, Lac S, Nigri J, Secq V, Rubis M, Bressy C, Serge A, Lavaut MN, Dusetti N, et al. Cancer-associated fibroblast-derived annexin A6+ extracellular vesicles support pancreatic cancer aggressiveness. J Clin Invest. 2016;126:4140-56.

62. Phillips P. Pancreatic stellate cells and fibrosis. In: Pancreatic Cancer and Tumor Microenvironment. Edited by Grippo PJ, Munshi HG. Trivandrum (India); 2012.

63. Takikawa T, Masamune A, Yoshida N, Hamada S, Kogure T, Shimosegawa T. Exosomes derived from pancreatic stellate cells: MicroRNA signature and effects on pancreatic cancer cells. Pancreas. 2017:46:19-27.

64. Syn N, Wang L, Sethi G, Thiery JP, Goh BC. Exosome-mediated metastasis: from epithelial-mesenchymal transition to escape from immunosurveillance. Trends Pharmacol Sci. 2016;37:606-17.

65. Liu Y, Cao X. Organotropic metastasis: role of tumor exosomes. Cell Res. 2016;26:149-50

66. Wortzel I, Dror S, Kenific CM, Lyden D. Exosome-mediated metastasis: communication from a distance. Dev Cell. 2019;49:347-60.

67. Seo Y, Baba H, Fukuda T, Takashima M, Sugimachi K. High expression of vascular endothelial growth factor is associated with liver metastasis and a poor prognosis for patients with ductal pancreatic adenocarcinoma. Cancer. 2000;88:2239-45.

68. Wei D, Le X, Zheng L, Wang L, Frey JA, Gao AC, Peng Z, Huang S, Xiong $H Q$, Abbruzzese JL, Xie K. Stat3 activation regulates the expression of vascular endothelial growth factor and human pancreatic cancer angiogenesis and metastasis. Oncogene. 2003;22:319-29.

69. Wang L, Zhou W, Zhong Y, Huo Y, Fan P, Zhan S, Xiao J, Jin X, Gou S, Yin $T$, et al. Overexpression of $G$ protein-coupled receptor GPR87 promotes pancreatic cancer aggressiveness and activates NF-kappaB signaling pathway. Mol Cancer. 2017;16:61.

70. Kitamoto S, Yokoyama S, Higashi M, Yamada N, Takao S, Yonezawa S. MUC1 enhances hypoxia-driven angiogenesis through the regulation of multiple proangiogenic factors. Oncogene. 2013;32:4614-21.

71. Webber J, Steadman R, Mason MD, Tabi Z, Clayton A. Cancer exosomes trigger fibroblast to myofibroblast differentiation. Cancer Res. 2010;70:9621-30

72. Webber JP, Spary LK, Sanders AJ, Chowdhury R, Jiang WG, Steadman $\mathrm{R}$, Wymant J, Jones AT, Kynaston H, Mason MD, et al. Differentiation of tumour-promoting stromal myofibroblasts by cancer exosomes. Oncogene. 2015;34:290-302.

73. Gu J, Qian H, Shen L, Zhang X, Zhu W, Huang L, Yan Y, Mao F, Zhao C, Shi Y, Xu W. Gastric cancer exosomes trigger differentiation of umbilical cord derived mesenchymal stem cells to carcinoma-associated fibroblasts through TGF-beta/Smad pathway. PLoS ONE. 2012;7:e52465.

74 Mulcahy LA, Pink RC, Carter DR. Routes and mechanisms of extracellular vesicle uptake. J Extracell Vesicles. 2014;3:1.

75. Li TD, Zhang R, Chen H, Huang ZP, Ye X, Wang H, Deng AM, Kong JL. An ultrasensitive polydopamine bi-functionalized SERS immunoassay for exosome-based diagnosis and classification of pancreatic cancer. Chem Sci. 2018;9:5372-82.

76. Pang Y, Wang C, Lu L, Wang C, Sun Z, Xiao R. Dual-SERS biosensor for one-step detection of microRNAs in exosome and residual plasma of blood samples for diagnosing pancreatic cancer. Biosens Bioelectron. 2019;130:204-13.

77. Williams C, Royo F, Aizpurua-Olaizola O, Pazos R, Boons GJ, Reichardt NC, Falcon-Perez JM. Glycosylation of extracellular vesicles: current knowledge, tools and clinical perspectives. J Extracell Vesicles. 2018;7:1442985.

78. Yokose T, Kabe Y, Matsuda A, Kitago M, Matsuda S, Hirai M, Nakagawa T, Masugi Y, Hishiki T, Nakamura Y, et al. O-glycan-altered extracellular vesicles: a specific serum marker elevated in pancreatic cancer. Cancers (Basel). 2020:12:1

79. Matsuda A, Kuno A, Yoshida M, Wagatsuma T, Sato T, Miyagishi M, Zhao J, Suematsu M, Kabe Y, Narimatsu H. Comparative glycomic analysis of exosome subpopulations derived from pancreatic cancer cell lines. J Proteome Res. 2020;19:2516-24.

80. Choi Y, Park U, Koo H, Park J, Lee D, Kim K, Choi J. Exosome-mediated diagnosis of pancreatic cancer using lectin-conjugated nanoparticles bound to selective glycans. Biosens Bioelectron. 2021;177:112980.

81. Boelens MC, Wu TJ, Nabet BY, Xu B, Qiu Y, Yoon T, Azzam DJ, TwymanSaint Victor C, Wiemann BZ, Ishwaran $\mathrm{H}$, et al. Exosome transfer from stromal to breast cancer cells regulates therapy resistance pathways. Cell. 2014:159:499-513.

82. Peinado H, Aleckovic M, Lavotshkin S, Matei I, Costa-Silva B, MorenoBueno G, Herqueta-Redondo M, Williams C, Garcia-Santos G, Ghajar $C$, et al. Melanoma exosomes educate bone marrow progenitor cells toward a pro-metastatic phenotype through MET. Nat Med. 2012:18:883-91.

83. Taylor DD, Gercel-Taylor C. Exosomes/microvesicles: mediators of cancer-associated immunosuppressive microenvironments. Semin Immunopathol. 2011;33:441-54.

84. Shao H, Chung J, Balaj L, Charest A, Bigner DD, Carter BS, Hochberg $\mathrm{FH}$, Breakefield XO, Weissleder R, Lee H. Protein typing of circulating microvesicles allows real-time monitoring of glioblastoma therapy. Nat Med. 2012;18:1835-40.

85. Xu Y, Xu X, Gin A, Nshimiyimana J, Mooers B, Caputi M, Hannafon B, Ding W. SRSF1 regulates exosome microRNA enrichment in human cancer cells. Cell Commun Signal. 2020;18:130.

86. Yu W, Hurley J, Roberts D, Chakrabortty SK, Enderle D, Noerholm M, Breakefield XO, Skog JK. Exosome-based liquid biopsies in cancer: opportunities and challenges. Ann Oncol. 2021;32:466-77. 
87. Kamerkar S, LeBleu VS, Sugimoto H, Yang S, Ruivo CF, Melo SA, Lee JJ, Kalluri R. Exosomes facilitate therapeutic targeting of oncogenic KRAS in pancreatic cancer. Nature. 2017;546:498-503.

88. McAndrews KM, Xiao F, Chronopoulos A, LeBleu VS, Kugeratski FG, Kalluri R. Exosome-mediated delivery of CRISPR/Cas9 for targeting of oncogenic Kras(G12D) in pancreatic cancer. Life Sci Alliance. 2021;4:1.

89. King $\mathrm{H}$, Thillai $\mathrm{K}$, Whale $\mathrm{A}$, Arumugam $\mathrm{P}$, Eldaly $\mathrm{H}$, Kocher $\mathrm{HM}$, Wells $\mathrm{CM}$. PAK4 interacts with p85 alpha: implications for pancreatic cancer cell migration. Sci Rep. 2017;7:42575.

90. He LF, Xu HW, Chen M, Xian ZR, Wen XF, Chen MN, Du CW, Huang WH, Wu JD, Zhang GJ. Activated-PAK4 predicts worse prognosis in breast cancer and promotes tumorigenesis through activation of PI3K/AKT signaling. Oncotarget. 2017;8:17573-85.

91. Xu L, Faruqu F, Lim Y, Lim K, Liam-Or R, Walters A, Lavender P, Fear D, Wells C, Tzu-Wen Wang J, Al-Jamal K. Exosome-mediated RNAi of PAK4 prolongs survival of pancreatic cancer mouse model after loco-regional treatment. Biomaterials. 2021;264:120369.

92. Bhattacharya S, Pal K, Sharma A, Dutta S, Lau J, Yan I, Wang E, Elkhanany A, Alkharfy K, Sanyal A, et al. GAIP interacting protein C-terminus regulates autophagy and exosome biogenesis of pancreatic cancer through metabolic pathways. PLoS ONE. 2014;9:e1 14409.

93. Zhou W, Zhou Y, Chen X, Ning T, Chen H, Guo Q, Zhang Y, Liu P, Zhang Y, $\mathrm{LiC}$, et al. Pancreatic cancer-targeting exosomes for enhancing immunotherapy and reprogramming tumor microenvironment. Biomaterials. 2021;268:120546.

94. Batista IA, Melo SA. Exosomes and the future of immunotherapy in pancreatic cancer. Int J Mol Sci. 2019;20:1.

95. Xie Z, Gao Y, Ho C, Li L, Jin C, Wang X, Zou C, Mao Y, Wang X, Li Q, et al. Exosome-delivered CD44v6/C1QBP complex drives pancreatic cancer liver metastasis by promoting fibrotic liver microenvironment. Gut. 2021. https://doi.org/10.1136/gutjin-2020-323014.

96. Madhavan B, Yue S, Galli U, Rana S, Gross W, Müller M, Giese N, Kalthoff $H$, Becker T, Büchler M, Zöller M. Combined evaluation of a panel of protein and miRNA serum-exosome biomarkers for pancreatic cancer diagnosis increases sensitivity and specificity. Int J Cancer. 2015;136:2616-27.

97. Melo SA, Luecke LB, Kahlert C, Fernandez AF, Gammon ST, Kaye J, LeBleu VS, Mittendorf EA, Weitz J, Rahbari N, et al. Glypican-1 identifies cancer exosomes and detects early pancreatic cancer. Nature. 2015;523:177-82.

98. Lai X, Wang M, McElyea SD, Sherman S, House M, Korc M. A microRNA signature in circulating exosomes is superior to exosomal glypican-1 levels for diagnosing pancreatic cancer. Cancer Lett. 2017;393:86-93.

99. Herreros-Villanueva M, Bujanda L. Glypican-1 in exosomes as biomarker for early detection of pancreatic cancer. Ann Transl Med. 2016;4:64.

100. Machida T, Tomofuji T, Maruyama T, Yoneda T, Ekuni D, Azuma T, Miyai H, Mizuno H, Kato H, Tsutsumi K, et al. miR1246 and miR4644 in salivary exosome as potential biomarkers for pancreatobiliary tract cancer. Oncol Rep. 2016;36:2375-81.

101. Li Z, Jiang P, Li J, Peng M, Zhao X, Zhang X, Chen K, Zhang Y, Liu H, Gan L, et al. Tumor-derived exosomal Inc-Sox2ot promotes EMT and stemness by acting as a ceRNA in pancreatic ductal adenocarcinoma. Oncogene. 2018;37:3822-38.

102. Yan $Q$, Yuan $W$, Sun $X$, Zhang $M$, Cen F, Zhou S, Wu W, Xu Y, Tong L, Ma $Z$. Asparaginyl endopeptidase enhances pancreatic ductal adenocarcinoma cell invasion in an exosome-dependent manner and correlates with poor prognosis. Int J Oncol. 2018;52:1651-60.

103. Kimura H, Yamamoto H, Harada T, Fumoto K, Osugi Y, Sada R, Maehara $\mathrm{N}$, Hikita $\mathrm{H}$, Mori S, Eguchi H, et al. CKAP4, a DKK1 receptor, is a biomarker in exosomes derived from pancreatic cancer and a molecular target for therapy. Clin Cancer Res. 2019;25:1936-47.

\section{Publisher's Note}

Springer Nature remains neutral with regard to jurisdictional claims in published maps and institutional affiliations.

Ready to submit your research? Choose BMC and benefit from:

- fast, convenient online submission

- thorough peer review by experienced researchers in your field

- rapid publication on acceptance

- support for research data, including large and complex data types

- gold Open Access which fosters wider collaboration and increased citations

- maximum visibility for your research: over $100 \mathrm{M}$ website views per year

At BMC, research is always in progress.

Learn more biomedcentral.com/submissions 